\title{
DIE GRÖSSE DES ZUSTANDSMINIMALEN LR(O)-ANALYSATORS
}

R.Kemp,Universität des Saarlandes, Saarbrücken

Die Analyse eines Wortes w, welches mittels einer LR(k)-Grammatik $G(/ 6 /)$ erzeugbar ist,läßt sich bekanntlich in linearer leit mittels eines deterministischen "push-down-transducer" $\operatorname{DPDT}(a)$ ( $\mathrm{LR}(\mathrm{k})-$ Analysator) durchführen, dessen Schaltwerk ein endicher Automat a ( $\operatorname{LR}(k)$-Automat) ist,welcher die Menge aller Anfangswörter bis zur ersten Reduktion (Handle) und die folgenden $k$ Terminalzeichen erkennt. Dabei treten die Endzustände (Reduce-Zustände) im LR(k)-Automaten getrennt auf,d.h.es fallen keine Endzustände in Zwischenzustände (ShiftZustände) und keine Endzustände zusammen. Im allgemeinen geht allerdings die rasche Analysezeit auf Kosten aufwendiger Analysatoren,d.h. die Zustandsmenge des $\mathrm{LR}(\mathrm{k})$-Automaten wird sehr groß.(vergl./3/). Aus diesem Grund ist man an Verfahren interessiert, welche die Zustandsmenge vermindern, ohne auf eine korrekte Fehlererkennung bei der Analyse zu verzichten.

In der vorliegenden Arbeit werden zunächst zwei Typen von LR(k)-Automaten definiert: Der "A-minimale LR( $k)$-Automat" und der "g-minimale LR( $k$ )-Automat". Beide definieren einen $L R(k)$-Analysator, welcher genau die von der gegebenen $L R(k)$-Grammatik G erzeugte Sprache $\mathscr{L}(G)$ analysiert.Der A-minimale LR(k)-Analysator,welcher als Schaltwerk den A-minimalen $\operatorname{LR}(k)$-Automaten besitzt, hat die Eigenschaft, daß die Entscheidung, ob das Wort in der erzeugten Sprache liegt oder nicht, in kijzester Zeit getroffen wird. Der $\mathfrak{g}$-minimale $L R(k)$-Analysator mit dem $g$-minimalen $\mathrm{LR}(\mathrm{k})$-Automaten als Schaltwerk trifft die gleiche Entscheidung,allerdings mit stärkerer Verzögerung, besitzt dagegen aber die geringste Anzahl von Zuständen unter allen Analysatoren, in deren Schaltwerk die Endzustände getrennt auftreten und die zu vorgegebenem Wort aus der Sprache $\mathscr{L}(G)$ den zugehörigen Ableitungsbaum bzgl.G liefern. Dabei arbeiten beide Analysatoren auf Wörtern der Sprache gleich schnell. Im wesentlichen stellt sich die Konstruktion des g-minimalen LR(k)-Automaten aus dem A-minimalen LR(k)-Automaten als Minimierung spezieller partieller endicher Automaten dar. Als bemerkenswertes Ergebnis erhält man, daß i.a.auch die Mächtigkeit der Zustandsmenge des g-minimalen LR(O)-Automaten exponentiell mit der Mächtigkeit des Hilfsalphabetes der gegebenen LR(O)-Grammatik wächst. 


\section{NOTATIONEN - DEETMITIONEN}

\section{Definition 1}

Ein 4-tupel $G=(I, T, P, \sigma)$ heiBt kontextfreie Grammatik (CFG) ïber dem Hilfsalphabet I, dem Endalphabet T,mit dem Produktionssystem $P$ und dem Axiom $\leftarrow(\$ I)$.Jede Regel $f_{i} \in P, 1 \leqslant i \leqslant \#$, ist von der Gestalt:

$$
f_{i}: Q\left(f_{i}\right) \longrightarrow Z\left(\hat{i}_{i}\right)
$$

mit $Q\left(f_{1}\right)=\sigma, Q\left(f_{j}\right) \in I, Z\left(f_{i}\right) \in(I \cup T)^{*}, 1 \leqslant i \leqslant \# P, j \geqslant 2, Q(f)$ heibt Quelle der Regel $f$ und $Z(f)$ heiBt $\underline{Z}$ iel der Regel $f$.

\section{Bemerkung 1}

Wie in / $/$ beschrieben, läßt sich jeder CFG eine freie $\mathrm{X}$-Kategorie $F(P, I U T)$ mit der Morphismenmenge MOR(F(P,IUT)) und dem freien Erzeugendensystem $P$ zuordnen.Jedem aus dem Axiom $\sigma$ mittels der $f \in P$ ableitbaren Wort $w$ entspricht damit ein Vorphismus $g \in \operatorname{MOR}(F(P, I \cup T))$ mit $Q(g)=\sigma$ und $Z(g)=w$, wobei $Q(g)$ bzw.Z(g) Quelle und $Z$ iel von g darstellen.

\section{Definition 2}

Ist $G=(I, T, P, \sigma)$ eine $C F G$, dann ist

$$
\gamma(G):=\left\{w \in(I \cup T)^{*} \mid(\exists g \in \operatorname{MOR}(F(P, I \cup T)))(Q(g)=G \wedge Z(g)=w\}\right.
$$

die Menge aller aus dem Axiom $\sigma$ ableitbaren Satzformen. Die Menge $\mathscr{L}(G):=\gamma(G) \cap T^{*}$ ist die von der CFG erzeugte kontextfreie sprache.

\section{Definition 3}

Eine CFG $G=(I, T, P, G)$ heibt chomskyreduziert (CCFG), falls gilt:

(i) $(\forall A \in I)(\exists f \in P)\left(Q(f)=A \wedge Z(f) \in \mathbb{T}^{*}\right)$

(ii) $(\forall A \in I)\left(\exists g \in \operatorname{MOR}(\mathcal{F}(P, I \cup T)) \wedge \exists u, v \in(I \cup T)^{*}\right)(Q(g)=\sigma \wedge z(g)=u A v)$

\section{Definition 4}

Ist $G=(I, T, P, \sigma)$ eine $\operatorname{CCFG}$ und $g \in \operatorname{MOR}(F(P, I \cup T)$, dann heibt

$$
g=\left(1_{u_{m}} \times f_{m} \times 1_{v_{m}}\right) \circ \ldots o\left(1_{u_{1}} \times f_{1} \times 1_{v_{A}}\right)
$$

mit $u_{i}, v_{i} \in(I \cup T)^{*}, f_{i} \in P, 1 \leqslant 1 \leqslant m$, sequentielle Darstellung von g. Besitzt $g$ genau eine sequentielle Darstellung, dann ist $g$ total unzerlegbar. Eine sequentielle Darstellung heibt antikanonisch, wenn $I\left(v_{i}\right)<1\left(v_{i+1}\right)+1$, $1 \leqslant i \leqslant m-1$, gilt. Dabei ist $I(w)$ die Länge des Wortes w. Wenn es $z u$ g eine antikanonische Darstellung obiger Form mit $Q(g)=\sigma$ gibt, dann heibt $\left(1_{u_{m}} \times f_{m} \times 1_{v_{m}}\right)$ H-Faktor von $g$.

Definition 5

Ist $G=(I, T, P, \sigma)$ eine $C C F G$, dann sei mit $f \in P$ die Menge $R(f)$ wie folgt 
definiert:

$$
\begin{aligned}
& R(f):=\left\{w Z(f) \mid\left(\exists v \in(I \cup T)^{*} \unlhd g \in \operatorname{MOR}(\mathcal{F}(P, I \cup T))(Q(g)=\sigma \wedge g \text { total unzer- }\right.\right. \\
& \text { legbar } \left.\left.\wedge\left(1_{\mathrm{W}} \mathrm{x} \in \mathrm{x}{ }_{\mathrm{v}}\right) \text { H-Faktor von } \mathrm{g}\right)\right\}
\end{aligned}
$$

Die Menge $R(P)$ mit

$$
R(P):=\underbrace{}_{f \in P} R(f)
$$

heißt Menge der regulären Anfangswörter.

Definition 6

Ein endicher Automat (EA) ist ein 7 -tupel $a=(\varepsilon, \mathcal{f}, \mathcal{f}, \alpha, \sigma, \delta, \lambda)$ mit

(i) $\varepsilon, \rho, f, \theta$ sind endliche Mengen (Eingabezeichen, Zwischenzustände,

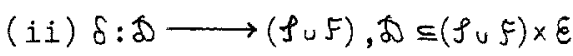
Endzustände, Ausgabezeichen)

(iii) $\lambda: \mathcal{H} \longrightarrow \theta, \mathcal{H} \subseteq(\mathcal{H}, \mathcal{F}) \times \varepsilon$ (Uberfürungsfunition)

(iv) $\alpha \in \mathcal{\rho}$

(Ausgabefunktion)

(Startzustand)

Ein EA a heiBt

$$
\begin{aligned}
& \text { deterministisch }(D E A) \Longleftrightarrow(\forall(s, x) \in \Phi)(\# \delta(s, x) \leqslant 1) \\
& \text { vollständig }(\mathrm{VEA}) \longleftrightarrow D=\mathscr{X}=(\rho \cup F) \times \varepsilon \\
& \text { streng partiell (SPEA) } \longleftrightarrow \precsim=\mathscr{x}
\end{aligned}
$$

\section{Bemerkung 2}

Ist a ein deterministischer VEA, dann bezeichnen wir mit $\delta(\alpha, w)$ den $Z u-$ stand, in den man durch Lesen des Wortes $w$ vom Startzustand $\alpha$ aus gelangt.

\section{DER g-MINIMALE IR(O)-ANALYSATOR}

Beil der Analyse eines Wortes w, welches mittels einer IR(O)-Grammatik G erzengbar ist, liest man das Wort von links nach rechts und die Entscheidung, ob an der gerade gelesenen Stelle eine Reduktion f,d.h.eine Ersetzung von $Z(f)$ durch $Q(f)$, vorzunehmen ist oder nicht, kann eindeutig aus dem bereits gelesenen Teilwort getroffen werden. Da in der Menge der regulären Anfangswörter (1.Def.5) alle Anfangswörter bis zur ersten Reduktion aufgelistet sind,genugt bei der Analyse eines Wortes $w$ die Kenntnis der Menge $R(P)$. In / / wurde gezeigt,daB die Menge $R(P)$ für jede CCFG als homomorphes Bild eines Standardereignisses regulär ist und damit von einem endichen Automaten A erkannt wird.In $/ 2 /$ wurde die Berechnung der Überführungsfunktion $\delta$ dieses deterministischen VEA (DVEA) $A=(I \cup T, \mathcal{f},\{(f) \mid f \in P\}, \alpha, P \cup\{1, s\}, \delta, \lambda)$ effizient gestaltet und darüberhinaus gezeigt,daß dieser $\mathrm{VEA}$ im automatentheoretischen Sinne reduziert und damit minimal ist. Ist $w=u x v \in(I \cup T)^{*}, u v \in(I \cup T)^{*}, x \in(I \cup T)$, 
dann gilt für die Überführungsfunktion $\delta$ und die Ausgabefunktion $\lambda$ :

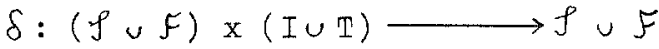

$$
\delta(z, x)= \begin{cases}(f) & \text { falls } u x \in R(f), f \in P \\ Z^{\prime} & \text { falls }\left(\exists y \in(I \cup T)^{*}\right)(u x y \in R(P)) \\ q & \text { sonst }\end{cases}
$$

und

$$
\begin{aligned}
\lambda:(\rho \cup f) \times(I \cup T) & \longrightarrow P \cup\{1, s\} \\
\lambda(z, x) & =\left\{\begin{array}{lll}
f & \text { falls } & \delta(z, x)=P \\
1 & \text { falls } & \delta(z, x) \in \mathcal{S},\{q\} \\
s & \text { falls } & \delta(z, x)=q
\end{array}\right.
\end{aligned}
$$

Dabei ist $q$ ein ausgezeichneter Zustand, in den man im Falle w $\notin f(G)$ gelangt.

Der Automat A besitzt bei vorliegender LR(O)-Grammatik die Eigenschaft, daß keine Endzustände zusammen- und keine Endzustände in Zwischenzustände fallen.Die Aequivalenz dieser Aussage mit dem Vorliegen einer $\mathrm{LR}(0)$-Grammatik ist in $/ 1 /, / 2 /$ gezeigt und gibt AnlaB zu folgender

Definition 7

Eine CCFG ist genau dann vom Typ $\operatorname{LR}(0)$, wenn fur alle $f^{\prime} f^{\prime} \in P, f \neq f^{\prime}$ gilt:

(i) $R(f) \cdot T^{*} \cap R\left(f^{\prime}\right)=\varnothing \quad$ und $\quad(i i) R(f) \cdot T^{+} \cap R\left(f^{\prime}\right)=\varnothing$

Es gilt der in $/ 5 \mathrm{a} /$ bewiesene

\section{SATZ 1}

Ist $G$ eine CCFG vom Typ IR(O), dann meldet der Analysator DPDT(A) mit dem endichen Automaten A als Schaltwerk bei dem Eingabewort w genau dann einen Analyseabbruch, wenn $w$ ein Anfangswort $w_{1}$ besitzt,zu dem keine Verlängerung $v$ zu einem aus $\sigma$ ableitbaren Wort existiert.

Damit besitzt der DPDT(A) die Eigenschaft,daß er zum frihest möglichen Zeitpunkt die Analyse abbricht.Wir bezeichnen ihn als A-minimalen IR(O)-Analysator der Sprache $\mathcal{L}(G)$. (Analysezeitminimal). Der Automat A heiBt A-minimaler $I R(0)$-Automat.

\section{REGULÄRE LR(O)-ÜBERDECKUNGEN}

Definition 8

Ist $G=(I, T, P, \tilde{S})$ eine $C C F G$ vom Typ $\operatorname{LR}(0)$ und A der zugehörige A-minimale $I R(O)$-Automat, welcher die reguläre Menge $R(P)$ erkennt, dann heiBt jedes Mengensystem $\hat{R}(P):=\{\hat{R}(f) \mid f \in P\}$ vermöge
(i) $(\forall f \in P)(\hat{R}(f)$ regulär $)$
(iii) $(\forall f \in P)\left(\hat{R}(f) \subseteq \hat{R}(f) \subseteq(I \cup T)^{*}\right)$
(ii) $(\forall f \in P)\left(\hat{R}(f) \cdot \mathbb{T}^{+} \cap \hat{R}(f)=\varnothing\right)$
(iv) $\left(\forall f, f^{\prime} \in P\right)\left(f \neq f^{\prime} \Longrightarrow \hat{R}\left(f^{\prime}\right) \cdot T^{*} \cap \hat{R}(f)=\varnothing\right)$
reguläre $I R(0)$-Überdeckung von $R(P)$. 
Es gilt der in $/ 5 \mathrm{a} /$ bewiesene

SATZ 2

Ist $G=(I, T, P, \sigma)$ eine CCFG vom Typ $\operatorname{LR}(0)$ und $\hat{R}(P)$ eine reguläre $L R(0)$ Überdeckung von $R(P)$, welche von dem $D E A ~ \hat{A}=(I u T, \hat{\jmath}, \xi, \hat{\alpha}, P \cup\{1, s\}, \hat{\delta}, \hat{\lambda})$ erkannt wird, dann stellt jeder DPDT $(\hat{A})$ ein Analysator der sprache $\mathcal{L}(G)$ dar.

\section{Bemerkung 3}

Iiefert der A-minimale $\operatorname{LR}(0)$-Automat A eine Reduktion $f$, dann ist diese auch anwendbar, d.h.der Automat gelangte durch Jesen von $z(f)$ in den Endzustand (f). Iiefert dagegen der Automat $\hat{A}$ eine Reduktion f, dann mu diese nicht notwendigerweise anwendbar sein, da $\hat{A}$ u. U.nur durch Jesen eines Endwortes von $Z(f)$ in den Endzustand (f) gelangte.Aus diesem Grund müssen die zu löschenden Kellerzeichen bei einer Ausführung einer Reduktion $f$ mittels des $\operatorname{DPDT}(\hat{A})$ mit den entsprechenden zeichen in $Z(f)$ auf Übereinstimmung verglichen werden.

\section{DER g-MINIMALE LR(O)-ANALYSATOR}

Ist $A$ der A-minimale $L R(O)$-Analysator bzgl.der $I R(O)$-Grammatik $G$ und $\hat{A}$ ein EA, welcher eine reguläre IR(O)-Überdeckung $\hat{R}(P)$ erkennt, dann bezeichnen wir mit. $\mathrm{A} z w . \hat{\hat{A}}$ diejenigen SPEA, welche durch Elimination des ausgezeichneten Zustandes q aus $A$ bzw. $\hat{A}$ entstehen,d.h. z.B.

$$
\frac{A}{P}=(I \cup T, \rho \backslash\{q\}, f, \alpha, P \cup\{1\}, \delta /(\rho,\{q\}) x(I \cup T), \lambda /(\rho \backslash\{q\}) \times(I \cup T))
$$

Falls keine Verwechslungen auftreten, setzen wir kinftig wieder:

$$
\frac{A}{P}=(I \vee T, \rho, F, \alpha, P \cup\{1\}, \delta, \lambda) \quad \text { und } \quad \hat{A}=(\operatorname{IvT}, \hat{\rho}, \mathcal{F}, \hat{\alpha}, P \cup\{1\}, \hat{\delta}, \hat{\lambda})
$$

Es gilt der in $/ 5 \mathrm{a} /$ gezeigte

SATZ 3

Es existiert $i . a$. kein Automatenhomomorphismus $\Phi$ mit

$$
\begin{array}{lll}
\text { (i) } \Phi(A)=\hat{A} & \text { bzw. } & \text { (ii) } \Phi(\hat{A})=\hat{A}
\end{array}
$$

Die Menge aller regulärer $L R(O)$-Überdeckungen von $R(P)$ bezeichnen wir mit $\hat{\mathscr{R}}(\mathrm{P})$. $\hat{\mathbb{R}}(\mathrm{P})$ ist die Menge aller EA $\hat{\mathrm{A}}$, welche $\hat{R}(P) \in \hat{\mathscr{R}}(\mathrm{P})$ erkennen. Wir geben nun die

Definition 9

Ist $G=(I, T, P, \sigma)$ eine CCFG vom Typ $\operatorname{LR}(0)$, dann heißt ein Analysator $\operatorname{DPDT}(\underset{\mathrm{A}}{\mathrm{P}})$ vermöge $\# \bar{\rho}=$ $\mathrm{NI} N \# \hat{\rho}$ $\hat{A} \in \hat{A}(P)$

$\hat{g}$-minimaler $I R(0)$-Analysator. (Zustandsminimal). Der Automat $\frac{\bar{A}}{\mathrm{P}}$ heißt $\partial=$ minimaler $\operatorname{IR}(0)$-Automat. 
Wie man leicht einsieht, erhält man jeden EA $\frac{\hat{A}}{P} \in \hat{\mathscr{C}}(P)$ aus $\frac{A}{P}$ durch geeignete Identifikation von Zuständen. Es dürfen solche Zustände $z, z^{\prime} \in \mathcal{f}$ nicht identifiziert werden, für die gilt:

$\left(\exists v \in(I \cup T)^{*}\right)(\delta(\eta, v)=(f) \wedge \delta(\xi, v) \in(\mathcal{S}, F) \backslash\{(\mathcal{f})\}), \eta, \xi \in\left\{z, z^{\prime}\right\}, \quad \eta \neq \xi$ da ansonsten der Endzustand (f) in einen Zwischenzustand oder einen von (f) verschiedenen Endzustand fallen würde.Zustände, welche nicht identifizierbar sind, d.h.der angegebenen Bedingung genügen, bezeichnen wir küftig als inkompatibel (ansonsten:kompatibel). Ein Minimierungsalgorithmus zur Konstruktion des g-minimalen Analysators $\overline{\mathrm{A}}$ ist in /5b/angegeben.

\section{DIE GRÖSSE DES Y-MINIMALEN IR(O)-ANALYSATORS $\bar{A}$}

Wir wollen nun einige Aussagen über die Mächtigkeit der Zustandsmenge $\bar{f} u f$ machen. Hierzu geben wir die

\section{Definition 10}

Ist $G=(I, T, P, \widetilde{T})$ eine CCFG, dann ist

$$
F(f, X):=\left\{f \in P \mid Z(f) \in(I \cup T)^{*} X, X \in(I \cup T)\right\}
$$

die Menge der $\underline{X-f i n a l e n ~ R e g e l n}$ und

$$
\mathbb{M}(f, X):=\left\{f \in P \mid Z(f) \in(I \cup T)^{*} \cdot X \cdot(I \cup T)^{+} \wedge(\exists g \in P)(g \in F(f, X))\right\}
$$

die Menge des $\mathrm{X}$-medialen Regeln.

Es gilt der

SATZ 4

Ist $G=(I, T, P, \sigma)$ eine CCFG vom Typ $\operatorname{LR}(0)$, dann gilt für die Mächtigkeit der Zustandsmenge $\bar{\rho}_{u} f$ eines $g$-minimalen $\operatorname{IR}(0)$-Automaten ${ }_{P}^{A}$ :

$$
\#(\rho \cup \mathcal{F}) \geqslant \# \mathbb{P}+\underset{X \in(I \cup T)}{A} X\left(\# F(f, X)-\delta_{O, \# M(f, X)}+1\right)
$$

Dabei ist $\delta_{i, k}$ das "Kronecker Symbol".

\section{Beweisskizze:}

Betrachte im A-minimalen $\operatorname{LR}(0)$-Automaten A alle Zustände, in die man durch Lesen eines $x \in\left(\right.$ I $T$ ) gelangt. Seien diese o.B.d.A. ( $f_{1}, \ldots, f_{k}, s_{1}$, . $\ldots, s_{m}$ mit $f_{i} \in \mathcal{F}$ und $s_{j} \in \mathcal{\rho}, 1 \leqslant i \leqslant k, 1 \leqslant j \leqslant$ m. Es gilt:
a) Jedes
(i)
b) Jedes
$s_{j}$
$\in F$ rith
ührt von einer $X$-finalen Regel

Für jedes $f_{i} \in \mathcal{F}$ muß im Automaten $\bar{A}$ mindestens ein Zwischenzustand $\bar{z}_{i} \in \bar{\rho}$ mit $\bar{\delta}\left(\bar{z}_{i}, X\right)=f_{i}$ existieren,d.h.mindestens \# $F(f, X)$ Zustände. Ungünstigenfalls sind die Zustände $s_{j} \in \mathcal{\rho}$ paarweise kompatibel und werden in $\bar{A} z u$ einem Zustand $\bar{s} \in \overline{\mathcal{S}}$ identifiziert, was einen weiteren $Z u-$ stand $s$ mit $\bar{\delta}(s, X)=\bar{s}$ erfordert. Damit folgt unmittelbar die Behauptung. 
Eine unmittelbare Folgerung von SATZ 4 ist das

\section{KOROLLAR}

Ist $G=(I, T, P, \tilde{\sigma})$ eine CCFG vom Typ $\operatorname{LR}(0)$ und endet jedes $Z(f), f \in P$, mit einem anderen Buchstaben $X \in$ (IUT), welcher nicht als Teilwort eines $Z\left(f^{\prime}\right), f^{\prime} \in P$, auftritt, dann gilt:

$$
\# \rho_{u} F \geqslant \# P+1
$$

\section{Definition 11}

Sei $G=(I, T, P, \sigma)$ eine $C C F G$ und $\varphi \in P(I)$ eine Hilfsalphabetkombination (HK).Wir definieren folgende Mengen:

$$
\begin{aligned}
& \{f\}_{\mathrm{A}}:=\{f \in P \mid Q(f)=A\} \quad \text { Bezeichnung: Regelpaket (RP) } \\
& \{f\}_{\varphi}:=\varliminf_{A \in \varphi}\{f\}_{A} \quad \text { Bezeichnung: Regelpaketkombination } \\
& V_{A}:=\left\{w \in(I \cup T)^{+} \mid\left(\exists f \in\{f\}_{A}\right)\left(Z(f)=w u \wedge u(I \cup T)^{*}\right)\right.
\end{aligned}
$$

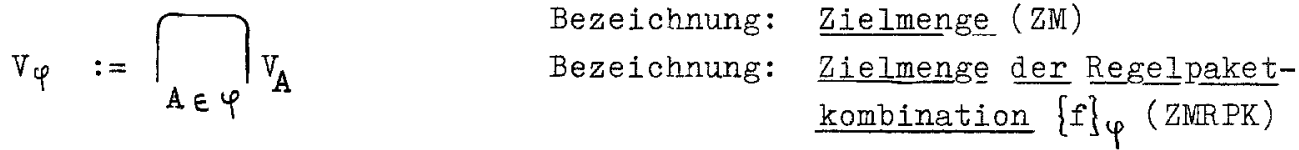

Wie in /2/,/3/ gezeigt, werden bei der Konstruktion des A-minimalen $\mathrm{LR}(0)$-Automaten sukzessive HK erzeugt, deren Gesamtheit wir mit $\mathbb{T}$ bezeichnen.Jedes $\varphi \in \mathbb{T}$ erzeugt gerade \#V $V_{\varphi}$ Zustände.Mit $i \in \mathbb{N}$ sei

$$
\prod_{i}:=\{\varphi \in \mathbb{T} \mid \# \varphi=i\}
$$

Es gilt nun der $/ 3 /, / 5 \mathrm{~b} /$ bewiesene

\section{SATZ 5}

Ist $G=(I, T, P, \sigma)$ eine CCFG vom Typ $\operatorname{LR}(0)$, dann gilt fur die Zustandsmenge $\rho \cup f$ des A-minimalen $\mathrm{IR}(0)$-Automaten ${ }_{\mathrm{P}}^{A}$ :

$\begin{aligned} \# \mathcal{I}_{\cup} \mathcal{F} & =1+\sum_{\varphi \in \pi} \# V_{\varphi}+\sum_{A \in I \cdot \pi}\left[\# V_{A}-\# \sum_{\varphi \in H_{A}} V_{\varphi}\right] \leqslant \\ & \leqslant 1+\sum_{A \in I} \# V_{A}+\sum_{i=1}^{m} \varphi\left(x_{i}\right) \leqslant 1+\sum_{A \in I} \# V_{A}+m \cdot\left(2^{\# I-1}-\# I\right)\end{aligned}$ mit $\mathcal{H}_{\mathrm{A}}:=\left(\mathbb{T} \backslash \mathbb{T}_{1}\right) \cap(R(I) \backslash R(I \backslash\{A\})), \varphi\left(x_{i}\right):=\left\{\varphi \in \mathbb{T} \backslash \mathbb{T}_{1} \mid x_{i} \in V_{\varphi}\right\}, 1 \leqslant i \leqslant m$ $\bigcup_{\varphi \in \mathbb{N} \mathbb{N}_{1}} V_{\varphi}:=\left\{x_{i} \mid 1 \leqslant i \leqslant m\right\}$

\section{Definition 12}

Ist $\mathbb{M}$ eine endiche Menge und $\mathbb{N} \subseteq R(M)$ mit

(i) $\left(\forall \varphi, \varphi^{\prime} \in \mathbb{N}\right)\left(\varphi \neq \varphi^{\prime} \wedge \varphi^{\prime} \neq \varphi\right)$

(ii) $\left(\forall \varphi, \varphi^{\prime} \in \mathbb{N}\right)(\exists\{A, B\})\left(\forall \varphi^{\prime \prime} \in \mathbb{N}\right)\left(A \in \varphi^{\prime} \varphi^{\prime} \wedge B \in \varphi^{\prime} \backslash \varphi \wedge\{A, B\} \neq \varphi^{\prime \prime}\right)$

dann heiBt $\mathbb{N}$ reduziertes Spernersystem (RSS). Ist nur (i) erfullt, dann bezeichnen wir $\mathrm{N}$ in Anlehnung an /7/ als Spernersystem (SS). 
Mit

sei

$$
\begin{aligned}
& \theta_{\mathrm{RSS}}:=\{\Phi \subseteq R(\mathrm{M}) \mid \Phi \text { ist RSS }\} \\
& \mathrm{F}_{\mathrm{RSS}}: \mathbb{N} \longrightarrow \mathbb{N}
\end{aligned}
$$

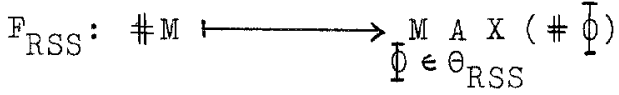

Es gilt der

\section{SATZ 6}

Ist $G=(I, \mathbb{T}, P, \sigma)$ eine $C C F G$ vom Typ $J R(O)$, dann gilt für die Zustandsmenge $\mathcal{J} \cup \mathcal{F}$ eines $\mathcal{z}$-minimalen $\operatorname{LR}(0)$-Automaten $\overline{\mathrm{A}}$ :

$$
\# \bar{\rho}_{\cup} f \leqslant 1+\sum_{A \in I} \# V_{A}+m \cdot F_{R S S}(\# I-1)
$$

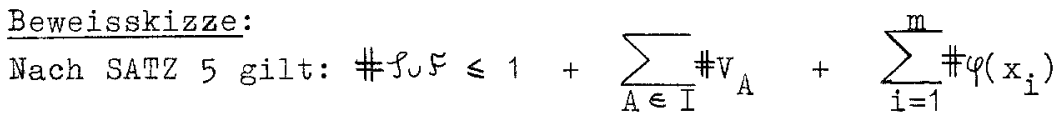

Betrachte für ein $i \in[1: m]$ die HK-en $\varphi\left(x_{i}\right):=\left\{\varphi_{1}, \varphi_{2}, \ldots, \varphi_{k}\right\}$ d.h. $\mathrm{x}_{i} \in \mathrm{V}_{\varphi_{j}}, 1 \leqslant j \leqslant k$

a) o.B.d.A.sei $\varphi_{1} \subseteq \varphi_{2} \subseteq \ldots \subseteq \varphi_{r}, r<k$

$\Longrightarrow\left(\forall A \in \sum_{i=1}^{r} V_{\varphi_{i}}\right)(\exists f \in P)\left(Q(f)=A \wedge Z(f)=x_{i} w\right)$

Diese Regeln erzeugen im Automaten A $r$ verschiedene Zustände.Da. $\varphi_{i} \subseteq \varphi_{r}, 1 \leqslant i \leqslant r-1$ und $\varphi_{r} \in \mathbb{T} \Longrightarrow$ Die Zustände sind kompatibel und können zu einem Zustand identifiziert werden.Dies gilt für alle Inklusionsketten in $\varphi\left(x_{i}\right)$, so daB $\varphi\left(x_{i}\right)$ höghstens soviele Zustände in $\bar{A}$ erzeugt,wie SS ỉber I möglich sind.

b) Nach den Identifizierungen in Teil a) ist $\varphi\left(x_{i}\right)$ zu $\psi\left(x_{i}\right)$ mit

$\left(\forall \varphi, \varphi^{\prime} \in \psi\left(x_{i}\right)\right)\left(\varphi \neq \varphi^{\prime} \wedge \varphi^{\prime} \ddagger \varphi\right)$ vermindert worden. Existiert in $\psi\left(x_{i}\right)$ eine Teilmenge $\eta$ mit

$\left(\exists \varphi, \varphi^{\prime} \in \eta\right)(\forall\{A, B\})\left(\exists \varphi^{\prime \prime} \in \eta\right)\left(A \in \varphi \backslash \varphi^{\prime} \wedge B \in \varphi^{\prime}, \varphi \wedge\{A, B\} \subseteq \varphi^{\prime \prime}\right)$ dann ist leicht einzusehen, daß die Zustände, welche durch $\varphi, \varphi^{\prime}$ erzeugt werden, kompatibel sind, da jedes $\{A, B\} \operatorname{mit} A \in \varphi \backslash \varphi^{\prime} \wedge B \in \varphi^{\prime}, \varphi$ in einem $\varphi$ " $\in \eta$ auftritt. Sukzessive Anwendung dieses Verfahrens ergibt, daß $\varphi\left(x_{1}\right)$ bzgl. des $\xi$-minimalen Automaten $\underset{p}{\bar{A}}$ höchstens soviele Zustände erzeugt, wie RSS über I möglich sind,d.h. $\psi\left(x_{i}\right)$ wird zu $\rho\left(x_{i}\right)$ mit \# $\rho\left(x_{i}\right) \leqslant F_{R S S}(\# I-1)$ vermindert.Damit folgt die Beh. Eine Untersuchung der Funktion $F_{\text {RSS }}$ liefert den

SATZ 7

Ist $\mathbb{M}$ eine enaliche Nenge mit \# $\mathbb{M} \geqslant 2$, dann gilt: $F_{R S S}(\# M)>\sqrt{2} \# \mathbb{M}$

Bemerkung 4

Ist $G=(I, T, P, \sigma)$ eine $C C F G$ und $\varphi=\left\{A_{1}, \ldots, A_{s}\right\} \in R(I)$, dann sei

$$
f_{i}: Q\left(f_{i}\right) \longrightarrow w \varphi=Z\left(f_{i}\right), 1 \leqslant i \leqslant s
$$


die Menge der Produktionen

$$
f_{i}: Q\left(f_{i}\right) \longrightarrow \mathrm{WA}_{i} \quad 1 \leqslant i \leqslant s
$$

Es gilt der

\section{SATZ 8}

Die Wächtigkeit der Zustandsmenge des $y$-minimalen Automaten wächst i.a. exponentiell mit der Mächtigkeit des Hilfsalphabetes der zugrunde liegenden CCFG vom Typ $\operatorname{LR}(0)$.

Beweisskizze:

(a)Betrachte folgende Grammatikfolge $G_{2 n}:=\left(I_{2 n}, T_{2 n}, P_{2 n}, \sigma\right)$ mit

$$
\begin{array}{ll}
I_{2 n}:=\left\{A_{i} \mid 1 \leqslant i \leqslant 2 n\right\} \quad & T_{2 n}:=\{a\} \cup X \cup Y \text { mit } \\
& X:=\left\{x_{i} \mid 1 \leqslant i \leqslant 2^{n}\right\} \\
& Y:=\left\{y_{i} \mid 1 \leqslant i \leqslant n\right\} \\
& X \cap Y=\varnothing, a \notin X \cup Y
\end{array}
$$

$$
\begin{array}{lll}
f_{\& n+1} & : \sigma \longrightarrow x_{\&+1} \varphi_{\&+1} & 0 \leqslant \& \leqslant 2^{n}-1,1 \leqslant i \leqslant n \\
f_{n 2^{n}+j} & : \sigma \longrightarrow y_{j} & 1 \leqslant j \leqslant n \\
f_{n\left(2^{n}+1\right)+2 B-1} & : A_{B} \longrightarrow A_{B} & 1 \leqslant B \leqslant 2 n \\
f_{n\left(2^{n}+1\right)+2 I} & : A_{B} \longrightarrow y\left[\frac{1+1}{2}\right] & 1 \leqslant 1 \leqslant 2 n
\end{array}
$$

wobei gilt:

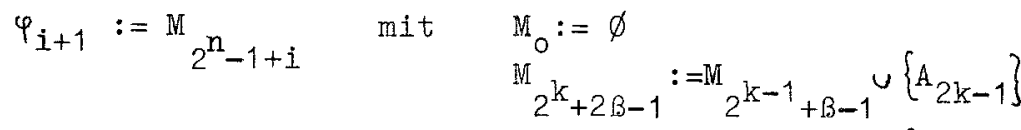

$$
\begin{aligned}
& \mathrm{M}^{\mathrm{k}}+2 B \quad:=\mathrm{M} 2^{\mathrm{k}-1}+B-1=\left\{A_{2 k}\right\} \\
& 0 \leqslant k \leqslant n, 0 \leqslant B \leqslant 2^{k-1}
\end{aligned}
$$

Es gilt:

$$
\mathscr{L}\left(G_{2 n}\right)=X a^{*} Y \cup Y
$$

Man zeigt nun folgende Aussagen:

(b)Mit $\Phi:=\varphi_{1} \cup \varphi_{2} \cup \ldots \cup \varphi_{2}$ und $y \in Y$ gilt:

$$
\left(\forall \varphi_{1}, \varphi_{2} \in \Phi\right)\left(\exists A_{1} \in \varphi_{1}\right)\left(\exists A_{2} \in \varphi_{2}\right)\left(A_{1} \neq A_{2} \Rightarrow\left\{A_{1} \rightarrow y, A_{2} \rightarrow y\right\} \subseteq P_{2 n}\right)
$$

(c) $\pi=\{\sigma\} \cup\left\{\varphi_{i} \mid 1 \leqslant i \leqslant 2^{n}\right\}$

(d) Jede CCFG $G_{2 n}$ ist vom Typ $\operatorname{LR}(0)$.

(e)Alle Zustände im A-minimalen IR(O)-Automaten sind inkompatibel.

(f)Der A-minimale IJR(O)-Automat und der g-minimale IR(O)-Automat sind isomorph.

(g) Mit SATZ 5 folgt: $\neq \bar{\rho} \cup F=(n+2) 2^{n}+5 n+1$

$$
=\left(\# I_{2 n} / 2+2\right) \cdot \sqrt{2^{\# I_{2 n}}}+5 \cdot \# I_{2 n} / 2+1
$$


6. SCHLUSSBENERKUNGEN

(a) Der - -minimale IR(0)-Automat besitzt die gleiche Eigenschaft wie der A-minimale LR(O)-Automat, nämlich daß zur Reduktionsentscheidung keine Berechnung von "look-aheads" notwendig ist.Fordert man diese Eigenschaft der IR(k)-Invarianz nicht mehr, dann kann die Zustandsmenge noch weiter unter die Zustandsmenge des Automaten $\bar{A}$ vermindert werden. (vergl./4/,/5b/)

(b) Nach Bemerkung 3 wird die Anwendbarkeit einer Reduktion auf dem Kellerbana des DPDT $(\bar{A})$ getroffen. Der definierte Kompatibilitätsbegriff von Zuständen kann leicht dahingehend verallgemeinert werden, daß gewisse Endzustände im Automaten ${\underset{P}{A}}_{\text {a }}$ zusamenfallen, ohne die Analyse uber linear anwachsen zu lassen.Es dürfen solche Endzustände nicht zusammenfallen, denen Regeln $f, f^{\prime} \in P, f \neq f^{\prime}$, mit $z(f)=w Z\left(f^{\prime}\right)$ entsprechen, da in diesem Fall die Entscheidung zwischen $f$ und $f^{\prime}$ auf dem Kellerband i.a. nicht mehr getroffen werden kann.

(c) Eine Minimierung des A-minimalen LR( $\mathrm{k}$ )-Automaten f zum $\mathrm{g}$-minimalen LR( $k$ )-Automaten mit $k>1$, kann auf die gleiche Art und Weise wie fiur den Fall $k=0$ durchgeführt werden. (vergl./5b/)

\section{LITERATUR}

$/ 1 /$ G.Hotz-V.Claus

$/ 2 /$ R.Kemp

/3/ R.Kemp

$/ 4 /$ R.Kemp

$/ 5 a /, / 5 b / R \cdot K e m p$

/6/ D.Knuth

/7/ E.Sperner
"Automatentheorie und formale Sprachen"

III. Formale Sprachen, BI.Hochschulskripten

(1971), 823a

"IR(k)-Analysatoren", Dissertation

Saarbruicken (1973)

"An Estimation of the set of States of the minimal $L R(0)$-Acceptor"

Automata, Languages and Programming(M.Nivat.ed) North Holland Publishing Co, Amsterdam (1973) $563-574$

"Die GröBe des minimalen Analysators einer kontextfreien Grammatik"

Lecture Notes in Ec.and Math.Syst.,78, (1972) 99-106

"Minimierung von $\operatorname{LR}(\mathrm{k})$-Analysatoren" $(1,2)$ Berichte des Math. Inst., Saarbrücken (A75/1,2) "On the Translation of Languages from Left to Right" , Inf.and Control 8,(1965),607-639 "Ein Satz über Untermengen einer endichen Menge" ,Math.Zeitsch. 27 (1928) .544-548 